\title{
Frecuencia de uso de las TIC y evaluación del perfil de competencias digitales en estudiantes de educación
}

\author{
Roque Vladimir Carrión Ramos \\ roque.carrion@upch.pe \\ https://orcid.org/0000-0002-2299-9260 \\ Universidad Nacional Mayor de San Marcos \\ Lima- Perú
}

\section{RESUMEN}

La investigación buscó determinar la relación que existe entre las variables frecuencia de uso de las TIC y el perfil de competencias digitales en los estudiantes del décimo semestre 2019-II de la Escuela Profesional de Educación de la Universidad Nacional Mayor de San Marcos. La investigación fue de enfoque cuantitativo, nivel descriptivo correlacional, diseño no experimental transversal. Se administraron los instrumentos CUTIC y CDAES. La muestra de estudio estuvo conformada por 153 estudiantes de las especialidades de inicial, primaria y secundaria. Los resultados encontrados concluyen que no existe relación entre las variables del estudio.

Palabras clave: TIC; competencia digital; funcionamiento y conceptos de las tecnologías avanzadas; residente digital. 


\title{
Frequency of use of ICT and evaluation of the profile of digital competences in Education students
}

\begin{abstract}
The research sought to determine the relationship between the varibles frequency of ICT use and the profile of digital competencies in the students of the tenth semester 2019-II of the Professional School of Education of the Universidad Nacional Mayor de San Marcos. The research was of quantitative approach, correlational descriptive level, nonexperimental cross-sectional design. The CUTIC and CDAES instruments were administered. The study sample consisted of 153 students from kindergarten, elementary and high school. The results found conclude that there is no relationship between the variables of the study.
\end{abstract}

Keywords: ICT; digital competence; operation and concepts of advanced technologies; digital resident.

Artículo recibido: 10. Junio. 2021 Aceptado para publicación: 16. Julio. 2021 Correspondencia: roque.carrion@upch.pe Conflictos de Interés: Ninguna que declarar 


\section{INTRODUCCIÓN}

En el siglo XX, las Tecnologías de la Información y la Comunicación (TIC) emergieron con fuerza dentro de la sociedad. Para Adell (1997), Trahtemberg (2000), Monereo y Pozo (2001), Magro et al. (2014), su inmersión en la cotidianidad fue lo más llamativo a las variaciones asociadas a la era de la Información, han cambiado el modo de trabajar, de entretenimiento, de socializar, de comportarse, de pensar y de aprender. Castells (1996) afirma que la sociedad decide su destino de acuerdo a su capacidad de apropiarse y controlar la tecnología. Esto obliga a la necesidad de identificar la frecuencia de uso de las TIC por parte de los estudiantes de Educación, qué dispositivos utilizan, cuánto tiempo le destinan, para qué actividades lo usan, qué sienten cuando dejan de usarlas. Además, la presencia de las TIC exige la adquisición de nuevas competencias, las competencias digitales, las cuales les permitirán darle un uso y aprovechamiento adecuado a las tecnologías avanzadas, preparándolos para los retos del presente siglo.

Las TIC, así como las competencias digitales, están en proceso de construcción, tanto en sus conceptualizaciones como en sus dimensiones. En la opinión de Baelo y Cantón (2009), las TIC han recibido diversas denominaciones, como nuevas tecnologías, tecnologías avanzadas, nuevas tecnologías de la información y la comunicación, que en el fondo engloban lo mismo; Chacón (2007) señala que las denominaciones se dieron de forma temporal y no considerando las características de las herramientas y/o recursos tecnológicos.

Las TIC son aquellas que incluyen a las tecnologías que permiten que la información, comunicación y el conocimiento puedan ser procesados, transmitidos, manipulados, almacenados y recuperados de una manera rápida y segura. Agrupa a las computadoras, tabletas, teléfonos inteligentes, Internet, programas informáticos y aplicaciones. Los usos de las TIC son diversos, sirviendo para conseguir información, de entretenimiento, de herramienta de comunicación, para aprender, entre otros.

Jiménez, Alvarado y Llopis (2017), señalan que la frecuencia de uso de las TIC está en aumento, tanto para el entretenimiento como la formación profesional, haciéndose difícil medir el uso que le dan los estudiantes universitarios, así como la dependencia que puede generar en ellos. La Frecuencia de uso de las TIC es la cantidad de tiempo -en horas- que se destina al día para la manipulación y aprovechamiento de las TIC en el entretenimiento, comunicación, juegos y aprendizaje; sobre este aspecto, Area, Hernández y Sosa (2016) 
sugieren medir la frecuencia de uso de las TIC en tres niveles: baja frecuencia, media frecuencia y alta frecuencia. Para Flores, López y Rodríguez (2016), consideran una baja en la frecuencia de uso de las TIC, si se da menos de dos horas al día; una media, si el tiempo está entre 2 a 4 horas; se considera una alta frecuencia, cuando se destina el tiempo a más de 4 horas al día. Las dimensiones de esta variable son frecuencia de uso de las TIC para el entretenimiento, mensajes y red social - beneficio de las TIC en el ámbito educativo- conducta y emoción que producen las TIC.

Sobre la competencia digital, en la opinión de Carrera y Coiduras (2012), no hay una definición universal; Esteve (2015) plantea que el concepto es polisémico y con múltiples matices, existiendo muchas conceptualizaciones. Para Pech y Prieto (2016) y Avitia y Uriarte (2017), está en camino de construcción. De Pablos, Colás, Conde y Reyes (2016) señalan la dificultad para definirla y que sea aceptada por la comunidad científica en su totalidad. Carrera y Coiduras (2012) afirman que la competencia digital tiene sinónimos equivalentes como competencia TIC, Informacional, Tecnológica, Estándares TIC; todas estas giran en torno del aprovechamiento de las tecnologías avanzadas para el aprendizaje y el desempeño laboral o profesional.

La competencia digital es aquella que agrupa los conocimientos, procedimientos y actitudes que permiten usar y aprovechar las TIC de manera crítica, segura y creativa, tanto en su formación profesional como en el entretenimiento, basándose en el uso apropiado de las computadoras, los teléfonos móviles inteligentes, las aplicaciones y programas de computadora para la investigación, la comunicación, la colaboración, la producción de material y el óptimo actuar en el mundo digital. Para Carrión (2020), el perfil de competencia digital de los estudiantes, se divide en tres niveles: nivel básico (en inicio de adquisición), nivel intermedio (en proceso), nivel avanzado (logrado).

Las dimensiones del perfil de competencias digitales del presente estudio, se basaron de los Estándares de Tecnologías de la Información y la Comunicación (NETS) de la International Society for Technology in Education (ISTE, 2008), el Marco Común de Competencia Digital Docente - DIGCOMP del INTEF (2017), DIGCOMP: A Framework for Developing and Understanding Digital Competence in Europe (Ferrari, 2013), Plan de Alfabetización Tecnológica de Extremadura - AUPEX (2017), DIGCOMP: A Framework for Developing and Understanding Digital Competence in Europe del Centro de Investigaciones Comunes de la Comisión Europea - European 
Commission's Joint Research Centre - Unión Europea (Carretero et al. 2017); siendo las siguientes: funcionamiento y concepto de las tecnologías avanzadas, acceso y uso de la información, comunicación y colaboración a través de tecnologías digitales, residente digital, resolución de problemas, creación de contenidos digitales.

En Latinoamérica, las investigaciones acerca este tema son escasas, el estudio para esclarecer sobre este tema, busca diagnosticar el nivel de frecuencia de uso de las TIC y de las competencias digitales que poseen los estudiantes, así como determinar la relación entre las variables del estudio. Es imprescindible conocer si los estudiantes de Educación están adquiriendo, sobre la base de la frecuencia de uso de las TIC, un perfil óptimo de las competencias digitales, y no quedar sumergidos en un analfabetismo digital.

\section{ESTRATEGIAS METODOLÓGICAS O MATERIALES Y MÉTODOS}

Teniendo en cuenta a Hernández et al. (2014), el estudio fue de enfoque cuantitativo, empleó la estadística, puso a prueba las hipótesis y expresó los resultados en valores numéricos, tipo básica, nivel descriptivo correlacional, diseño no experimental, transversal. El estudio empleó la prueba estadística Ji cuadrado de Pearson para contrastar la relación entre la frecuencia de uso de las TIC (variable 1) y el perfil de competencias digitales (variable 2), así como la relación entre la variable 1 con cada una de las dimensiones de la variables 2; si el valor obtenido es menor o igual que el nivel de significación, $\leq .05$, existe asociación o dependencia entre las variables; por el contrario, si el valor alcanzado es mayor al nivel de significancia, > .05, existe independencia entre las variables.

La población estuvo constituida por estudiantes del décimo ciclo de la Escuela profesional de Educación de la Universidad Nacional Mayor de San Marcos, matriculados en el semestre académico 2019-II, conformada por 252 estudiantes. Para seleccionar la muestra se aplicó la fórmula para la ecuación estadística para proporciones poblacionales, con un margen del 5\%, un nivel de confianza del $95 \%$ y considerando una población de 252 personas. La muestra se determinó empleando la siguiente fórmula:

$$
\mathrm{n}=\frac{\mathrm{z}^{2}(\mathrm{p} \times \mathrm{q})}{\mathrm{e}^{2}+\frac{\left(\mathrm{z}^{2}(\mathrm{p} \times \mathrm{q})\right)}{\mathrm{N}}}
$$

La muestra estuvo conformada por 153 estudiantes, se aplicó el instrumento a estudiantes entre las edades de 21 a 30 años, donde 103 pertenecieron al sexo femenino y 50 al masculino. 
El estudio empleó el Cuestionario para evaluar la Frecuencia de uso de las Tecnologías de la Información y la Comunicación - CUTIC elaborado por Jiménez et al. (2017), consta de 28 reactivos, agrupados en tres dimensiones, las escalas y valores del instrumento fueron: $0=$ Nunca, $1=$ Raramente, $2=$ Ocasionalmente, $3=$ Frecuentemente, 4 = Muy frecuentemente. Además, se aplicó el Cuestionario para el diagnóstico de la Competencia Digital del estudiante de Educación Superior - CDAES elaborado por Gutiérrez y Cabero (2016), consta de 44 reactivos, agrupados en seis dimensiones, las escalas y valores del instrumento son del 1 al 10: el valor 1 es sinónimo de no realizar lo que se exige; en cambio, el 10 indica el dominio completo de lo exigido. La validez de los instrumentos fue calculada a través del Coeficiente V de Aiken, CUTIC alcanzó un valor de 0,95, mientras que el CDAES un 0,94. La confiabilidad de ambos instrumentos fue calculada por medio del Alfa de Cronbach, el CUTIC obtuvo un valor de 0,87, mientras que el CDAES un 0,92.

\section{RESULTADOS Y DISCUSIÓN}

Antes de pasar al análisis es conveniente realizar una descripción de las variables generales de los encuestados, la muestra de estudiantes, divididos por sexo, correspondiendo al femenino 103 (67.3\%) y al masculino 50 (32.7\%). Las edades de los estudiantes, las cuales se repartieron de la siguiente manera, de 21-25 años, 142 (92.8\%) y de 26-30 años, $11(7.2 \%)$.

La figura 1 resume las frecuencias por cada dimensión de la variable Frecuencia de uso de las TIC.

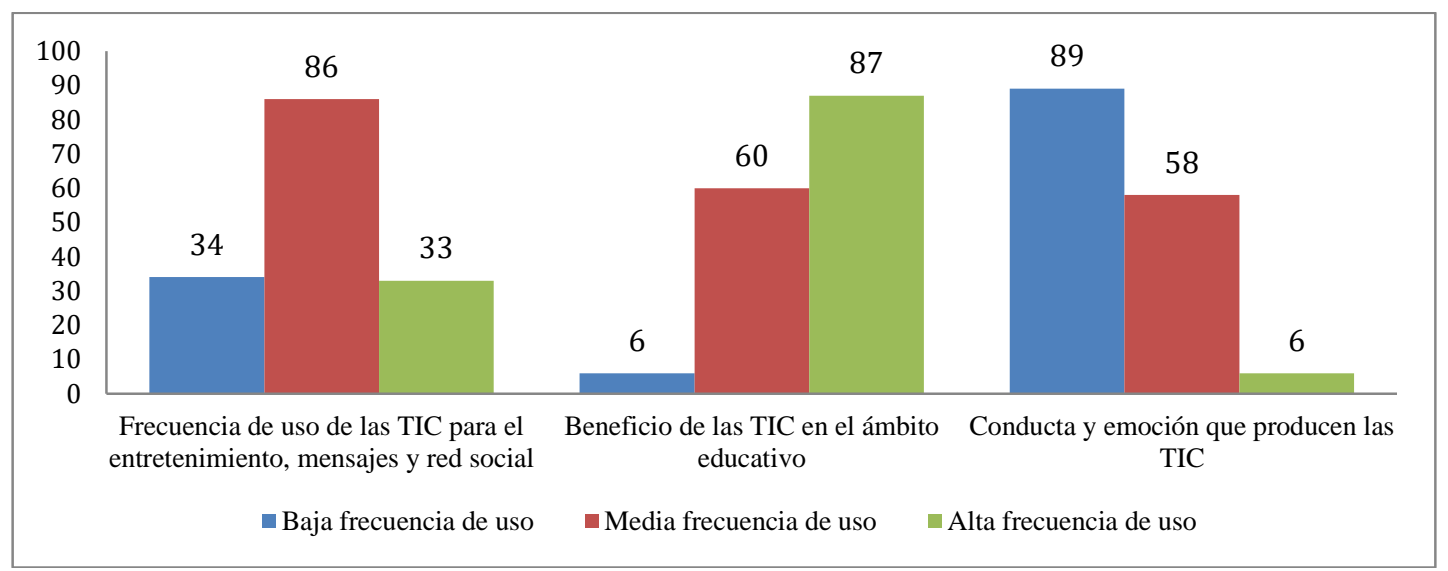

Figura 1. Frecuencias por cada dimensión de la Frecuencia de uso de las TIC.

La figura 2 resume los porcentajes por cada dimensión de la variable Frecuencia de uso de las TIC. 


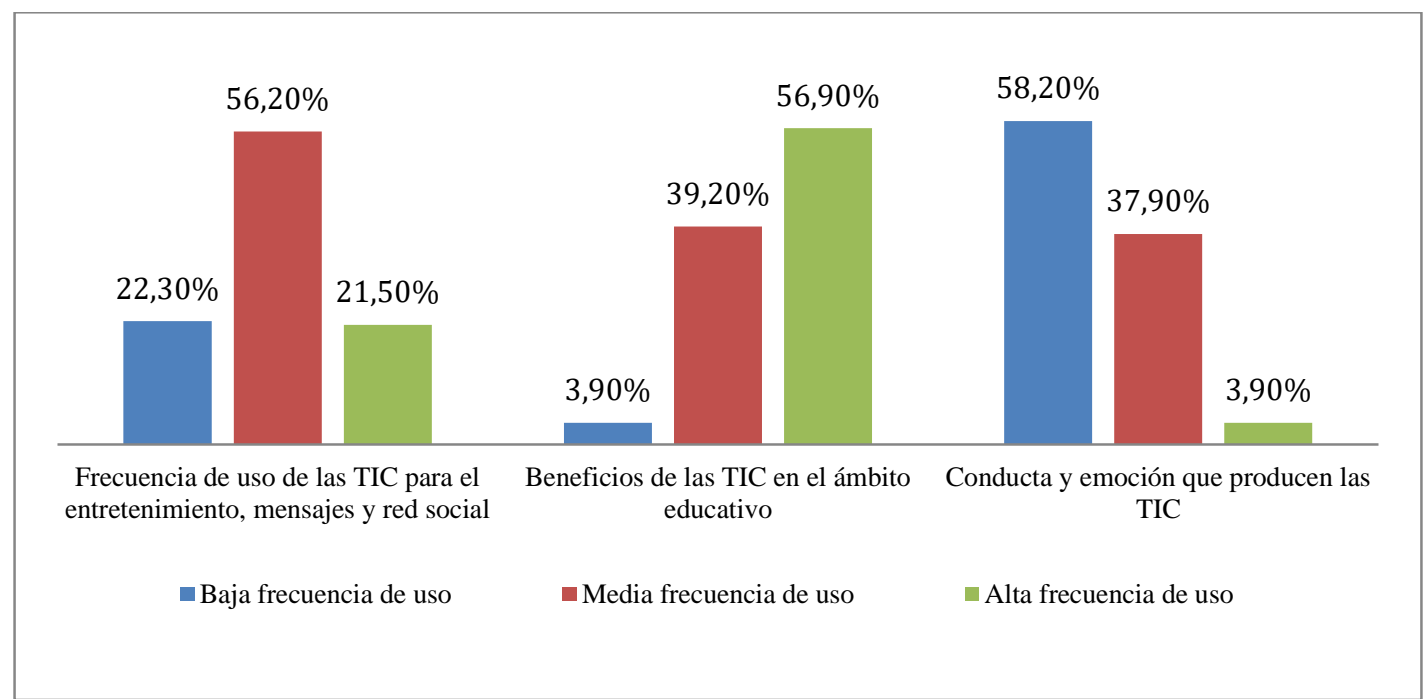

Figura 2. Porcentajes por cada dimensión de la Frecuencia de uso de las TIC.

La figura 3 resume las frecuencias por cada dimensión de la variable Perfil de competencias digitales.

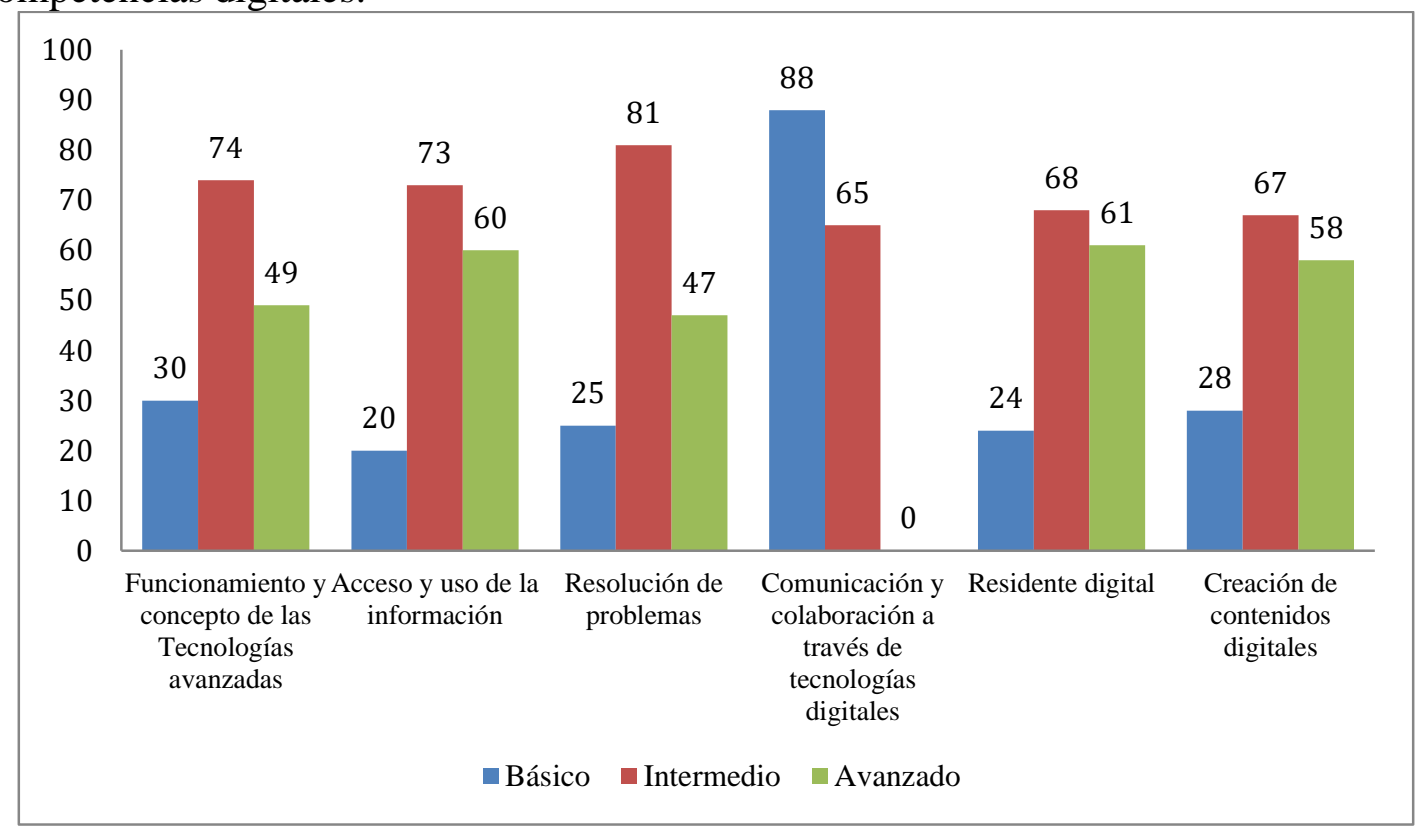

Figura 3. Frecuencias por cada dimensión del Perfil de competencias digitales.

La figura 4 resume los porcentajes por cada dimensión de la variable Perfil de competencias digitales. 


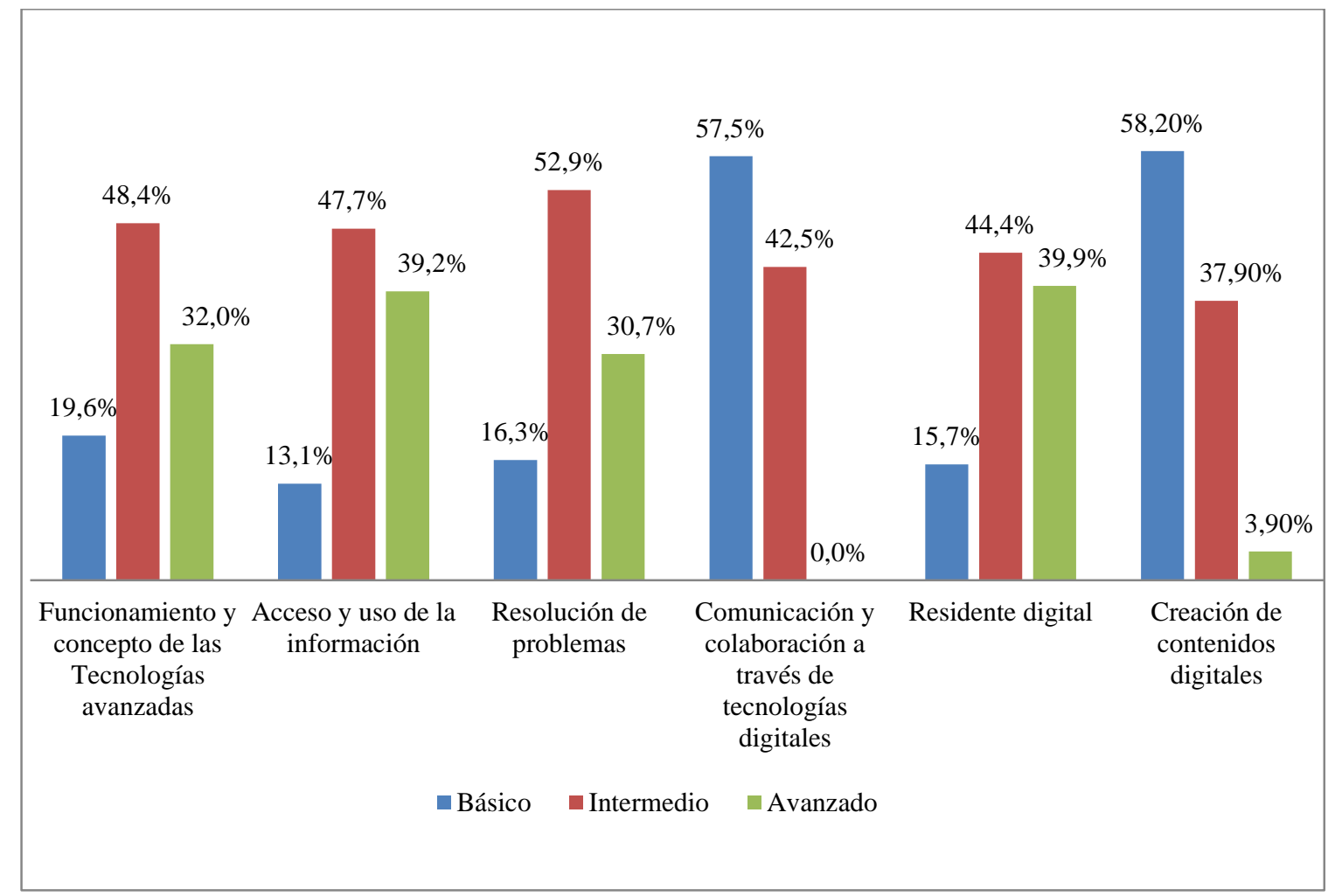

Figura 4. Porcentajes por cada dimensión del perfil de competencias digitales.

En lo referente al nivel de la Frecuencia de uso de las TIC, en la tabla 1 se observa que la variable alcanzó una media frecuencia de uso que representa el 73.2\%, mientras que la baja frecuencia de uso obtuvo un $26.8 \%$.

Tabla 1: Frecuencias y porcentajes de la variable Frecuencia de uso de las TIC

\begin{tabular}{lcc}
\hline \multicolumn{1}{c}{ Puntajes } & $\mathrm{f}$ & $\%$ \\
\hline Baja frecuencia de uso & 41 & 26.8 \\
Media frecuencia de uso & 112 & 73.2 \\
Alta frecuencia de uso & 0 & 0 \\
Total & 153 & 100.0 \\
\hline
\end{tabular}

Estos resultados evidencian que en los estudiantes no existe una dependencia hacia las TIC, haciendo un uso equilibrado sin llegar a una ansiedad por usar sus dispositivos tecnológicos y conectarse a Internet (Jiménez et al. 2017). Es necesario señalar que una media frecuencia de uso tampoco asegura un uso adecuado de las TIC. Sobre este aspecto, Echeburúa y Corral (2010) señalan que no debe considerarse a la frecuencia media de uso de las TIC como una adicción; lo peligroso es el uso inútil de los dispositivos tecnológicos, que puede producir cambios en su conducta y vida cotidiana, donde 
pierden el control hacia un uso y aprovechamiento de las TIC para su formación profesional.

Asimismo, Gallardo et al. (2016), señalan que los estudiantes aprovechan las TIC, en especial sus dispositivos móviles, para navegar por Internet en la búsqueda de contenidos ajenos a su formación profesional, juegos en línea y conectarse a las redes sociales; es decir, las usan para el entretenimiento y fines sociales, el llamado ocio digital móvil lúdico - social, haciéndolos vulnerables para no aprovecharlas en el aprendizaje ni desenvolverse con éxito en el siglo XXI.

Las TIC deben ingresar a la vida universitaria como asignatura obligatoria, para mejorar la formación de los estudiantes y prepararlos para que sepan darle una frecuencia de uso productiva para el aprendizaje y así reducir el analfabetismo tecnológico. Stiglitz (2015) lo considera necesario para esta sociedad que se basa y desenvuelve con las TIC. Por su parte, Vivas, Andrés y Gómez (2016), así como Zempoalteca et al. (2017), señalan que el uso correcto permitirá a los estudiantes estar preparados ante situaciones impredecibles de su campo profesional, siendo necesario rediseñar los programas de formación inicial y continua.

En lo referente al perfil de competencias digitales, se observa en la tabla 2 que se obtiene un nivel intermedio de $53.6 \%$ que representa a más del $50 \%$ de la muestra en estudio.

Tabla 2: Frecuencias y porcentajes del Perfil de competencias digitales

\begin{tabular}{lcc}
\hline \multicolumn{1}{c}{ Puntajes } & f & \% \\
\hline Básico & 33 & 21.6 \\
Intermedio & 82 & 53.6 \\
Avanzado & 38 & 24.8 \\
Total & 153 & 100.0 \\
\hline
\end{tabular}

Estos resultados manifiestan que los estudiantes necesitan reforzar dichas competencias, estando en proceso de superar la fase de iniciación (Rangel y Peñalosa, 2013). Para Jiménez (2015), un nivel intermedio es sinónimo de no competente, situación preocupante, porque lo ideal es egresar con nivel avanzado. Por otra parte, López, Pozo, Morales y López (2019) indican que la formación inicial cumple un rol significativo para alcanzar ese nivel. Ríos (2016) y Solórzano (2007) señalan que la adquisición de competencias involucra una constante y creciente formación y perfeccionamiento 
profesional. Por su parte, Mena (2017), señala que el no acceso a Internet o carecer de dispositivos tecnológicos no son impedimentos para adquirir dichas competencias, para Castro (2017), esto se debe a que las herramientas tecnológicas no están siendo usadas para desarrollar dicha competencia, por otro lado, Zempoalteca et al. (2017) señalan que un nivel bajo de competencias digitales es indicador de la falta de un modelo pedagógico para desarrollarla, siendo necesario diseñar un perfil de egreso para los nuevos tiempos, como lo señala Area (2010b), mediante el cual, los estudiantes sean preparados para los desafíos del siglo XXI y sepan aprovechar todo lo que ofrece el entorno virtual.

Respecto a la relación entre la Frecuencia uso de las TIC y el perfil de Competencias digitales, el estadístico Ji cuadrado de Pearson obtuvo un valor sig. .139; el valor alcanzado es $>.05$, lo que indica que no existe dependencia o asociación entre ambas. Este resultado indica que la frecuencia en el uso de las TIC no es determinante, ni guarda relación o asociación para desarrollar las competencias digitales. Para Gallardo et al. (2016), el hecho de que los estudiantes usen las TIC no asegura que hayan adquirido las competencias digitales. Sobre este aspecto, De Pablos et al. (2016) sugieren que las investigaciones respecto a las tecnologías avanzadas deben estar dirigidas a determinar si el uso de las TIC está relacionado con las competencias digitales, siendo necesario que los datos obtenidos de esas investigaciones sean válidos y confiables, lo cual permitirá tomar decisiones de mejora, la información resultante podrá dirigir los programas curriculares de los estudiantes universitarios. Cózar y Roblizo (2014), Fernández, Leiva y López (2017), Islas y Franco (2018) señalan que los estudiantes deben ser conscientes de que el aprovechamiento en el empleo de las TIC les permitirá adquirir las competencias digitales o viceversa. Girón, Cózar y González (2019) indican la obligación de formar a los estudiantes en las competencias digitales, ya que el uso que le dan a las TIC es frecuente.

Según Suárez (2019), la competencia digital no se debe limitar a una destreza técnica que permita al estudiante manipular los dispositivos tecnológicos o navegar por Internet; involucra al desarrollo de dimensiones que le permitan saber comunicarse y colaborar, seleccionar de manera correcta la información de la web, solucionar problemas e inquietudes con la ayuda de las TIC, producir con la tecnología, así como cuidar la identidad digital. Todo esto llevará a un uso y aprovechamiento de las tecnologías, debe 
haber una integración entre las nuevas tecnologías, las competencias digitales y la educación, promoviendo la colaboración para su inserción de manera efectiva.

Respecto a la relación entre la Frecuencia uso de las TIC con cada una de las dimensiones del Perfil de competencia digitales, los resultados obtenidos luego de aplicar el estadístico Ji cuadrado de Pearson se detallan en la tabla 4.

Tabla 4: Correlación entre Frecuencia de las TIC y cada una de las dimensiones del Perfil de competencias digitales

\begin{tabular}{lccc}
\hline & $\begin{array}{c}\text { Frecuencia de uso } \\
\text { de las TIC }\end{array}$ & $\begin{array}{c}\text { Existe dependencia } \\
\text { o asociación }\end{array}$ \\
\hline Funcionamiento y conceptos de las & .000 & Sí \\
tecnologías avanzadas & & Sí \\
Acceso y uso de la información & .030 & Sí \\
Resolución de problemas & .000 & Sí \\
Comunicación y colaboración a través & .008 & Sí \\
de tecnologías digitales & & No \\
Residente digital & .036 & .096 & \\
Creación de contenidos digitales. &
\end{tabular}

En los resultados que muestra la tabla 4, se encontró que existe relación entre la frecuencia de uso de las TIC y el funcionamiento y concepto de las tecnologías avanzadas en los estudiantes, el estadístico Ji cuadrado obtuvo un valor sig .000 que es $<.05$. Sobre este aspecto, Peñalva, Napal y Mendioroz (2018) y Mendoza et al. (2015), concuerdan al señalar que es necesario desarrollar en el estudiante de Educación, el Funcionamiento y conceptos de tecnologías avanzadas; esto le permitirá aprovechar las TIC en su formación profesional, desenvolverse con éxito en la cultura digital y a la vez replicarlo a sus futuros estudiantes.

En los resultados que presenta la tabla 4, indica que existe relación entre la Frecuencia de uso de las TIC con el Acceso y uso de la información en los estudiantes, el estadístico Ji cuadrado obtuvo un valor sig .030 que es $<.05$. Sobre este aspecto, Cabero y Llorente (2008) consideran que las tecnologías brindan cantidad de información a los estudiantes, pero esto no asegura que estén bien informados, es necesario formarlos en la investigación y manejo de la información. Para De Pablos (2010), el uso de las TIC ejerce un rol fundamental en nuevas propuestas para la investigación. Gómez y Gutiérrez (2014), 
Arias, Torres y Yáñez et al. (2014) coinciden al señalar la necesidad de fomentar un uso adecuado de las TIC para evaluar la información existente en Internet, su pertinencia y veracidad; esto le permitirá al estudiante fortalecer esta dimensión.

En los resultados que detalla la tabla 4, señala que existe una relación entre la frecuencia de uso de las TIC y la resolución de problemas en los estudiantes de la Escuela Profesional de Educación, el estadístico Ji cuadrado obtuvo un valor sig .000 que es < .05. Cózar y Roblizo (2014), señalan que los estudiantes son conscientes de que el aprovechamiento en el empleo de las TIC, les ayudará adquirir las competencias digitales para resolver los problemas que se le presenten. A su vez, Gómez y Gutiérrez (2014) y Rodarte (2014) concuerdan al señalar la obligación de desarrollar esta dimensión, lo cual les permitirá a los estudiantes actuar como pensadores computacionales, usando las TIC para la investigación, siendo reflexivos y críticos. Sobre este aspecto, Gallego, Gámiz y Gutiérrez (2010) consideran que la formación de competencias digitales no debe quedar reducida a desarrollar destrezas tecnológicas, sino basarse en el conocimiento para la solución de problemas. Por su parte, Avello y López (2015) señalan que la incorporación de las TIC debe ser de manera crítica en todo momento.

En los resultados que presenta la tabla 4, indica una relación entre la frecuencia de uso de las TIC y la comunicación y colaboración a través de tecnologías digitales en los estudiantes, el estadístico Ji cuadrado obtuvo un valor sig .008 que es < .05. Aportan en este sentido, Area, Gutiérrez y Vidal (2012), quienes señalan que las TIC han ingresado con gran fuerza en la sociedad, que una vida no sería completa sin la comunicación por medio de la tecnología. Para Fundación Telefónica (2013), se debe ser cuidadoso con lo que se comunica y comparte, porque el anonimato no existe en Internet, quedando todo grabado, sumado a que el mensaje puede ser interpretado de manera errónea. Vallejos (2013) sugiere una formación en el uso de las TIC para la comunicación y colaboración. Sobre este aspecto, Esteve y Gisbert (2013) señalan que el aprovechamiento de las tecnologías avanzadas para la comunicación y colaboración, por parte de los estudiantes, es necesaria porque brinda una interacción síncrona y asíncrona por medio del chat, video conferencia, correo electrónico, etc., permite el uso de diversos dispositivos, como ordenadores y móviles, brinda distintas herramientas de comunicación como skype, whatsapp, etc., además permite la creación de comunidades virtuales de aprendizaje que comparten e intercambian intereses comunes y experiencias educativas, convirtiéndolos 
en productores y emisores de comunicación de mensajes educativos, dejando de ser receptores de mensajes.

En los resultados que detalla la tabla 4, se encontró una relación entre la Frecuencia de uso de las TIC y el Residente digital en los estudiantes, el estadístico Ji cuadrado obtuvo un valor sig .036 que es < .05. Sobre este aspecto, Vazquéz (2013) indica que la vida online o en línea de los estudiantes, debe ser responsable y saludable, para que sepan manejar su privacidad, reputación, seguridad e identidad digital; además, señala la necesidad de cuestionar e investigar cómo se está dando la formación de ellos respecto a las competencias digitales. Es necesario desarrollar la ciudadanía digital en los estudiantes de Educación, porque se formaría profesionales no consumistas ni pasivos, sino por el contrario, productores en la virtualidad y especialistas en pedagogía digital, que propiciarían en sus futuros estudiantes el correcto desenvolvimiento en el mundo digital a través de sus dispositivos tecnológicos, alejándolos de la dependencia inútil a los móviles, el ciberacoso y del peligroso sexting, adquiriendo la cuestión ética en el uso de las TIC.

En los resultados que muestra la tabla 4, señala que no existe una relación entre la frecuencia de uso de las TIC y la creación de contenidos digitales en estudiantes, el estadístico Ji cuadrado obtuvo un valor sig .96 que es > .05. Citando a Cabero y Llorente (2008), De Pablos (2010), Torres y Santa (2013), Zempoalteca et al. (2017) y Prendes,

Gutiérrez y Martínez (2018), es obligación de las facultades de Educación, orientar la innovación en la enseñanza y el aprendizaje mediante el uso de las TIC, de formarlos para que no sean simples consumidores o receptores de contenidos; por el contrario, que sean creadores de contenidos digítales e innoven con la tecnología, pasando de una cultura de consumir a una cultura de producir o crear, donde el empleo de las TIC sirva para innovar y mejorar la competencia digital de los estudiantes.

\section{CONCLUSIÓN O CONSIDERACIONES FINALES}

A partir de los datos estadísticos hallados en la investigación, se concluye que no existe relación entre las variables frecuencia de uso de las TIC y el perfil de competencias digitales en los estudiantes que participaron en el estudio. Esto exige diseñar programas de actualización, cursos y talleres que ayuden a la adquisición de un nivel avanzado en el perfil de competencias digitales y de un uso y/o manejo pertinente de las TIC en los 
docentes y estudiantes universitarios, lo que fortalecerá la empleabilidad laboral y preparación para el siglo XXI.

Según los resultados encontrados de la variable Frecuencia de uso de las TIC, indican que los estudiantes obtuvieron una media frecuencia de uso de las TIC, que representó el 73.2\%. La dimensión utilidad de las TIC en el ámbito educativo alcanzó una alta frecuencia, por otra parte, la dimensión conducta y emoción que producen las TIC logró una alta puntuación respecto a una baja frecuencia de uso de las TIC, lo que indica una escasa dependencia hacia las TIC por parte del estudiante. Se recomienda que se deben incentivar actividades de aprendizaje que fomenten a que la frecuencia de uso de las TIC esté destinada para ese fin; esto reducirá la frecuencia para el entretenimiento y ocio digital por parte de los estudiantes.

De los resultados encontrados de la variable perfil de competencias digitales, señalan que los estudiantes del décimo semestre 2019-II de la Escuela Profesional de Educación de la UNMSM, alcanzaron un nivel intermedio, que representó el 53.6\%, lo que indica que están camino a adquirir el nivel requerido de dichas competencias. La dimensión que obtuvo menor puntaje fue la de Comunicación y colaboración a través de las tecnologías digitales, lo que evidencia la poca capacidad para comunicarse y compartir información a través del uso de las TIC para su aprendizaje y enriquecimiento profesional. Se recomienda que los centros formadores de docentes, como las facultades de Educación y los Institutos pedagógicos, tengan la obligación de fomentar el uso adecuado y aprovechamiento de las TIC para que se alcance un nivel óptimo del perfil de competencias digitales, dotando los ambientes con el equipamiento tecnológico necesario para el aprendizaje de los estudiantes.

Los datos estadísticos recogidos evidencian que existe relación entre la frecuencia de uso de las TIC y el funcionamiento y concepto de las tecnologías avanzadas. Se infiere que el desarrollo de esta dimensión va a la par con la frecuencia de uso de las nuevas tecnologías que hacen los estudiantes de manera productiva en su formación profesional.

De los datos estadísticos recogidos, se evidencia la relación entre la frecuencia de uso de las TIC y el acceso y uso de la información. Se puede inferir que la frecuencia de uso pertinente de las TIC les permitirá ser competentes en la búsqueda y selección del conocimiento e información a través de las TIC. 
De los resultados hallados, se demuestra una relación entre la frecuencia de uso de las TIC y la resolución de problemas, lo que indica que un correcto uso de las TIC permitirá a los estudiantes a la solución de problemas apoyados en las tecnologías.

A partir de los datos estadísticos hallados, se evidencia la relación entre la frecuencia de uso de las TIC y la comunicación y colaboración a través de tecnologías digitales; esto nos indica la necesidad de fomentar en los estudiantes a que dominen y usen los ordenadores, dispositivos móviles, aplicaciones e Internet para comunicarse y compartir conocimiento, lo cual fortalecerá la dimensión.

Los resultados estadísticos, evidencian la relación entre la frecuencia de uso de las TIC y el residente digital. Se puede afirmar la necesidad de promover una frecuencia de uso de las TIC de modo responsable que ayude a fortalecer la convivencia en la residencia digital.

Los datos estadísticos recogidos, evidencian que no existe relación entre la frecuencia de uso de las TIC y la creación de contenidos digitales; por tanto, cabe señalar que una apropiada frecuencia de uso de las TIC destinada a la generación de ideas innovadoras en su formación profesional, les permitirá alcanzar un nivel aceptable de la dimensión.

Romani y Rivera (2019) indican que la sociedad actual necesita replantear la formación de los estudiantes de Educación, en busca de la adquisición de dicha competencia por parte de los estudiantes, el acelerado avance de la información y el conocimiento lo exige, siendo un reto del siglo XXI. Para Esteve (2015), las competencias digitales permitirán al estudiante a tener conocimiento de los ordenadores y dispositivos móviles, de conocer nuevas formas de aprender usando las nuevas tecnologías, de usar Internet para socializar profesionalmente y de convivir en el entorno virtual.

Esteve (2015), Gisbert, González y Esteve (2016), Leváno et al. (2019), consideran como obligación y reto formar a los estudiantes de Educación en las competencias digitales, ya que ellos son responsables de inculcar los saberes a las nuevas generaciones; Flores y Roig (2016) consideran que debe haber un replanteamiento de la formación universitaria, considerando a dichas competencias como relevantes para los egresados de Educación; Castellanos, Nieto y Parra (2018) consideran que debe haber seriedad en la formación de las competencias digitales. Por su parte, Peñalva et al. (2018) señalan que el nivel de los profesionales encargados de la educación debe ser alto; esto aseguraría a las nuevas generaciones estar preparadas para desenvolverse con éxito en la cultura digital. 


\section{LISTA DE REFERENCIAS}

Adell, J. (1997). Tendencias en educación en la sociedad de las tecnologías de la información. Edutec. Revista Electrónica de Tecnología Educativa, (7), 1-21. Recuperado de https://www.edutec.es/revista/index.php/edutece/article/view/570/299

Area, M., Gutiérrez, A. \& Vidal, F. (2012). Alfabetización digital y competencias informacionales. Barcelona: Ariel.

Area, M., Hernández, V., \&Sosa, J. (2016). Modelos de integración didáctica de las TIC en el aula. Comunicar: Revista científica iberoamericana de comunicación y educación, $24 \quad$ (47), 79-87. Recuperado de https://dialnet.unirioja.es/servlet/articulo?codigo $=5400275$

Arias, M., Torres, T. \& Yáñez, J. (2014) El desarrollo de competencias digitales en la educación superior. Historia y Comunicación Social, 19, 355-366. doi: http://dx.doi.org/10.5209/rev_HICS.2014.v19.44963

Avello, R. \& López, R. (2015). Alfabetización digital de los docentes de las escuelas de hotelería y turismo cubanas. Experiencias en su implementación. RUSC. Universities and Knowledge Society Journal, 12 (3), 3-15. doi:http://dx.doi.org/10.7238/rusc.v12i3.1994

Avitia, P. \& Uriarte, I. (2017). Evaluación de la habilidad digital de los estudiantes universitarios: estado de ingreso y potencial educativo. EDUTEC, Revista electrónica de Tecnología Educativa, (61). Recuperado de: http://dx.doi.org/10.21556/edutec.2018.61

Baelo, R., \& Cantón, I. (2009). Las tecnologías de la información y la comunicación en la educación superior. Estudio descriptivo y de revisión. Revista Iberoamericana de Educación, 50 (7), 1-12. doi: https://doi.org/10.35362/rie5071965

Cabero, J. \& Llorente, M. (2008). La alfabetización digital de los alumnos. Competencias digitales para el siglo XXI. Revista Portuguesa de Pedagogía, 42 (2), 7-28. (ISSN: 0870418).

Recuperado

de https://digitalisdsp.uc.pt/bitstream/10316.2/4673/3/La\%20alfabetizacion\%20digi tal\%20de\%2010s\%20alumnos.pdf 
Carrera, F. \& Coiduras, J. (2012). Identificación de la competencia digital del profesor universitario: un estudio exploratorio en el ámbito de las ciencias sociales. Revista de docencia universitaria, $10 \quad$ (2), 273- 298. doi: https://doi.org/10.4995/redu.2012.6108

Carretero,S., Vuorikari, R, \& Punie, Y. (2017). DigComp 2.1: The Digital Competence Framework for Citizens with eight proficiency levels and examples of use. Recuperado de http://europa.eu/!Yg77Dh

Castellanos, M., Nieto, Z. \& Parra, H. (2018) Interpretación de las competencias digitales profesorales en el contexto universitario. Revista Logos Ciencia \& Tecnología, 10 (1), 41-51. doi: 10.22335/rlct.v10i1.518

Castells, M. (1996). The Rise of the Network Society. Londres: Blackwell.

Castro, P. (2017).Competencias digitales para la alfabetización digital de los estudiantes de primer año de la carrera de turismo de la Universidad Mayor de San Andrés de la gestión 2016 (Tesis de maestría, Universidad Mayor de San Andrés, Facultad de Humanidades y Ciencias de la Educación. La Paz, Bolivia). Recuperado de https://repositorio.umsa.bo/xmlui/handle/123456789/10488

Chacón, A. (2007). La tecnología educativa en el marco de la didáctica. En J. Ortega, \& A. Chacón (coords.), Nuevas tecnologías para la educación en la era digital (pp. 25-42). Madrid: Pirámide.

Cózar, R. \& Roblizo, M. (2014). La competencia digital en la formación de los futuros maestros: percepciones de los alumnos de los Grados de Maestro de la Facultad de Educación de Albacete. RELATEC Revista Latinoamericana de Tecnología Educativa, 13 (2), 119- 133. ISSN 1695288X. doi: https://doi.org/10.17398/1695$\underline{\text { 288X.13.2.119 }}$

De Pablos, J. (2010). Universidad y sociedad del conocimiento. Las competencias informacionales y digitales. Revista de Universidad y Sociedad del Conocimiento, 7 (2), 6-16. $\quad$ Recuperado de http://openaccess.uoc.edu/webapps/o2/bitstream/10609/2601/2/Monografic_esp. pdf

De Pablo, J., Colás, P., Conde, J., \& Reyes, S. (2016). La competencia digital de los estudiantes de educación no universitaria: variables predictivas. BORDÓN. Revista de pedagogía, 69 (1), 1- 18. doi: 10.13042/Bordon.2016.48594. 
Echeburúa, E., \& De Corral, P. (2010). Adicción a las nuevas tecnologías ya las redes sociales en jóvenes: un nuevo reto. Adicciones, 22 (2), 91-96. Recuperado de http://www.adicciones.es/index.php/adicciones/article/view/196

Esteve, F. \& Gisbert, M. (2013). Competencia digital en la educación superior: instrumentos de evaluación y nuevos entornos. Enl@ce Revista Venezolana de Información, Tecnología y Conocimiento, 10 (3), 29-43. ISSN: 1690-7515. Recuperado de http://www.redalyc.org/articulo.oa?id=82329477003

Esteve, F. (2015). La competencia digital docente. Análisis de la autopercepción y evaluación del desempeño de los estudiantes universitarios de educación por medio de un entorno 3D (Tesis de doctorado, Universitat Rovira i Virgili. Tarragona, España). Recuperado de http://www.tdx.cat/bitstream/handle/10803/291441/tesis.pdf

Fernández, E., Leiva, J., \& López, E. (2017). Formación en competencias digitales en la universidad. Percepciones del alumnado. Campus Virtuales, 6 (2), 79-89. Recuperado de http://uajournals.com/ojs/index.php/campusvirtuales/article/view/249

Ferrari, A. (2013). DIGCOMP: A Framework for Developing and Understanding Digital Competence in Europe. Recuperado de https://publications.jrc.ec.europa.eu/repository/bitstream/JRC83167/lb-na26035-enn.pdf

Flores, C., \& Roig, R. (2016). Diseño y validación de una escala de autoevaluación de competencias digitales para estudiantes de pedagogía. Píxel-Bit. Revista de Medios $\quad y \quad$ Educación, 209-224. doi: https://doi.org/10.12795/pixelbit.2016.i48.14

Flores, K., López, M., \& Rodríguez, M. (2016). Evaluación de componentes de los cursos en línea desde la perspectiva del estudiante. Revista electrónica de investigación educativa, 18 23-38.Recuperado de http://redie.uabc.mx/redie/article/view/474

Fundación Telefónica (2013). Identidad Digital: El nuevo usuario en el mundo digital. Barcelona: Ariel. 
Gallardo, E., Marqués, L., Bullen, M., \& Strijbos, J. (2016). Hablemos de aprendices digitales en la era digital. Revista Mexicana de bachillerato a distancia, 8 (15), 148-182. doi: http://dx.doi.org/10.22201/cuaed.20074751e.2016.15.57385

Gallego, J., Gámiz, V., \& Gutiérrez, E. (2010). Competencias digitales en la formación del futuro docente. Propuestas didácticas. Trabajo de investigación. Universidad de Granada. Recuperado de https://idus.us.es/xmlui/bitstream/handle/11441/56115/competencias_digitales_e $\underline{\text { n_la_formacion_del_futuro_docente._propuestas_didacticas.pdf?sequence }=1}$

Girón, V., Cózar, R. \& González, J. (2019). Análisis de la autopercepción sobre el nivel de competencia digital docente en la formación inicial de maestros/as. Revista Electrónica Interuniversitaria de Formación del Profesorado, 22 (3), 193218.doi: https://doi.org/10.6018/reifop.373421

Gisbert, M., González, J., \& Esteve, F. (2016). Competencia digital y competencia digital docente: una panorámica sobre el estado de la cuestión. RIITE. Revista Interuniversitaria de Investigación en Tecnología Educativa, 0, 74-83. doi: https://doi.org/10.6018/riite2016/257631

Gómez, M. \& Gutiérrez, J. (2014). Competencia digital en estudiantes de Educación. Congreso Internacional EDUTEC - Noviembre 2014, El hoy y el mañana junto a las TIC. ISBN: 978-84-15881-91-9. Recuperado de https://idus.us.es/xmlui/bitstream/handle/11441/33307/Competencia_digital_en_ estudiantes_de_educacion.pdf?sequence=1\&isAllowed=y

Gutiérrez, J. \& Cabero, J. (2016). Estudio de caso sobre la autopercepción de la competencia digital del estudiante universitario de las titulaciones de grado de educación infantil y primaria. Profesorado. Revista de Currículum y Formación de Profesorado, 20 (2), 180-199. ISSN: 1138-414X Recuperado de http://www.redalyc.org/articulo.oa?id=56746946010

Hernández, R., Fernández, C., \& Baptista, P. (2014). Metodología de la investigación. 6ta ed. México: Mc Graw Hill.

Instituto Nacional de Tecnologías Educativas y de Formación del Profesorado (INTEF) (2017). Marco Común de Competencia Digital Docente. Proyecto. Ministerio de Educación Cultura y Deporte. Gobierno de España. Recuperado de 
http://educalab.es/documents/10180/12809/MarcoComunCompeDigiDoceV2.pd $\underline{\mathrm{f}}$

International Society for Technology in Education (ISTE) (2018). Iste standards for educators. Recuperado de https://www.iste.org/standards/for-educators

Islas, C., \& Franco, S. (2018). Detección de patrones en competencias digitales manifestadas por estudiantes universitarios. Edutec. Revista Electrónica de Tecnología Educativa, (64), 51-66. doi: http://dx.doi.org/10.21556/edutec.2018.64.1079

Jiménez, V., Alvarado, J. M., \& Llopis, C. (2017). Validación de un cuestionario diseñado para medir frecuencia y amplitud de uso de las TIC. EDUTEC. Revista Electrónica de Tecnología Educativa, 61. doi: https://doi.org/10.21556/edutec.2017.61

Jiménez, J. (2015). Estudio sobre los estándares TIC en educación en los futuros docentes de la Facultad de Educación de la Universidad Complutense de Madrid. (Tesis de doctorado, Universidad Complutense de Madrid. Madrid, España). Recuperado de https://eprints.ucm.es/30925/1/T36158.pdf

Lévano-Francia, L., Sanchez, S., Guillén-Aparicio, P., Tello-Cabello, S., Herrera-Paico, N., \& Collantes-Inga, Z. (2019). Competencias digitales y educación. Propósitos y Representaciones, 7 (2), 569-578. doi: http://dx.doi.org/10.20511/pyr2019.v7n2.329

López J., Pozo, S., Morales, M., \& López, E. (2019). Competencia digital de futuros docentes para efectuar un proceso de enseñanza y aprendizaje mediante realidad virtual. Edutec. Revista Electrónica De Tecnología Educativa, (67), 1-15. https://doi.org/10.21556/edutec.2019.67.1327.

Mena, F. (2017). La competencia digital en el grado de enfermería de la Universidad de Huelva. (Tesis de doctorado, Universidad de Huelva, Huelva, España) Recuperado de http://rabida.uhu.es/dspace/bitstream/handle/10272/14672/La_competencia_digit al.pdf? sequence $=4$

Mendoza, R., Enciso, R., Fonseca, M., \& González, S. (2015). Actitudes de los docentes: Incorporación de las tecnologías en la práctica docente. Revista EDUCATECONCIENCIA, 7 (8), 69-81. Recuperado de 
http://dspace.uan.mx:8080/xmlui/bitstream/handle/123456789/114/Actitudes\%2 0de\%20los\%20docentes\%20Incorporaci\%C3\%B3n\%20de\%20las\%20tecnolog \%C3\%ADas\%20en\%20la.pdf?sequence $=1 \&$ is Allowed $=\mathrm{y}$

Monereo, C., \& Pozo, J. (2001). ¿En qué siglo vive la escuela? El reto de la nueva cultura educativa. Cuadernos de pedagogía, (298), 50-55. Recuperado de http://redined.mecd.gob.es/xmlui/handle/11162/31050

Pech, S., \& Prieto, M. (2016). La medición de la Competencia Digital e Informacional. ResearchGate, 1-24. Recuperado de https://www.researchgate.net/profile/Manuel_Prieto3/publication/303075873_La _medicion_de_la_Competencia_Digital_e_Informacional/links/5736e97608ae29 8602e16bd4/La-medicion-de-la-Competencia-Digital-e Informacional.pdf?origin=publication_detail

Plan de Alfabetización Tecnológica de Extremadura - AUPEX (2017). Marco Europeo de Competencias Digitales para la Ciudadanía. Recuperado de https://www.nccextremadura.org/competenciadigital/

Peñalva, A., Napal, M., \& Mendioroz, A. (2018). Competencia digital y alfabetización digital de los adultos (profesorado y familias). International Journal of New Education, 1 (1), 1-13. doi: https://doi.org/10.24310/IJNE1.1.2018.4892

Prendes, M., Gutiérrez, I., \& Martínez, F. (2018). Competencia digital: una necesidad del profesorado universitario en el siglo XXI. RED. Revista de Educación a Distancia, (56), 1-22, Artíc. 7. doi: http://dx.doi.org/10.6018/red/56/7

Rangel, A., \& Peñalosa, E. (2013). Alfabetización digital en docentes de educación superior: construcción y prueba empírica de un instrumento de evaluación. PíxelBit. Revista de Medios y Educación, (43), 9-23. doi: http://dx.doi.org/10.12795/pixelbit.2013.i43.01

Rios, M. (2016). Enfoques de gestión educativa. Para no directivos. En el marco de la dinámica interna y la administración moderna. Lima: Editorial GP.

Rodarte, R. (2014). Uso de las TIC en los profesores de tiempo completo de la Licenciatura en Música de la Universidad Veracruzana. (Tesis de maestría, Universidad Veracruzana. Xalapa, México). Recuperado de https://cdigital.uv.mx/handle/123456789/42132 
Romani, U., \& Rivera, J. (2019). Empleabilidad de los docentes que aplican la estrategia metacafé lector en la Universidad Ricardo Palma. Scientia, 21 (21), 247-253. doi: https://doi.org/10.31381/scientia.v21i21.2791

Solórzano, J. (2007). El portal de paradigmas. Para organizaciones competitivas. (Lo deseable). Lima: Fondo Editorial UNMSM.

Stiglitz, J. (2015). La creación de una sociedad del aprendizaje. Una nueva aproximación al crecimiento, el desarrollo y el progreso social. Madrid: La Esfera de los Libros.

Suárez, C. (16 de septiembre 2019). No es lo mismo hablar de desarrollo educativo con tecnología que de inclusión tecnológica en educación [Entrevista a Cristóbal Suárez]. Recuperado de https://educared.fundaciontelefonica.com.pe/entrevistacristobal-suarez/

Torres, R., \& Santa, A. (2013). Construcción de Identidad Digital profesional de estudiantes y docentes. "SocialOVTT", una experiencia de innovación docente orientada a la empleabilidad. En: XI Jornadas de Redes de Investigación en Docencia Universitaria, Retos de futuro en la enseñanza superior. Universidad de Alicante. ISBN 978-84-695-8104-9. Recuperado de https://rua.ua.es/dspace/bitstream/10045/43257/1/2013-XI-Jornadas-Redes62.pdf

Trahtemberg, L. (2000). El impacto previsible de las nuevas tecnologías en la enseñanza y la organización escolar. Revista Iberoamericana de Educación, (24), 37- 62. Recuperado de http://rieoei.org/rie24a02.htm

Vallejos, E. (2013). El impacto de la implementación de las TIC en la Evaluación del Desempeño Laboral del docente universitario: Estudio de casos del uso de PAIDEIA por los docentes de la FGAD-PUCP en el período 2010-2011 (Tesis de maestría, Pontificia Universidad Católica del Perú. Lima, Perú). Recuperado de tesis.pucp.edu.pe/...VALLEJOS_MAMANI_ELIZABETH_IMPACTO_PAIDEIA. $p d f$

Vazquéz, A. (2013). Incidencia de la brecha digital en grupos de iguales a partir de la interactividad entre la identidad física y la identidad digital. Recuperado de http://eujournal.org/files/journals/1/books/AlfonsoVazquezAtochero.pdf

Vivas, M., Andrés, S., \& Gómez, M. (2016). Desarrollo de competencias digitales en docencia online: la asignatura Cimientos del curso de adaptación a grado en 
ingeniería de edificación. RED-Revista de Educación a Distancia, (49), 1-29. doi: http://dx.doi.org/10.6018/red/49/8

Zempoalteca, B., Barragán, J., González, J., \& Guzmán, T. (2017). Formación en TIC y competencia digital en la docencia en instituciones públicas de educación superior. Apertura, 9 (1), 80-96. ISSN 2007-1094. Recuperado de http://www.scielo.org.mx/scielo.php?script=sci_arttext\&pid=S1665-

61802017000200080 